\begin{tabular}{|c|l|}
\hline Title & Horo-tight spheres in Hyperbolic space \\
\hline Author(s) & Buosi, Marcelo; Izumiya, Shy uichi; Maria A parecida, Soares Ruas \\
\hline Citation & Hokkaido University Preprint Series in Mathematics, 932, 1-17 \\
\hline Issue Date & 2009-1-14 \\
\hline DOI & 10.14943/84080 \\
\hline Doc URL & http://hdl.handle.net/2115/69740 \\
\hline Type & bulletin (article) \\
\hline File Information & pre932.pdf \\
\hline
\end{tabular}

Instructions for use 


\title{
HORO-TIGHT SPHERES IN HYPERBOLIC SPACE
}

\author{
MARCELO BUOSI, SHYUICHI IZUMIYA, AND MARIA APARECIDA SOARES RUAS
}

\begin{abstract}
We study horo-tight immersions of manifolds into hyperbolic spaces. The main result gives several characterizations of horo-tightness of spheres, answering a question proposed by T. Cecil and P. Ryan. For instance, we prove that a sphere is horo-tight if and only if it is tight in the hyperbolic sense. For codimension bigger than one, it follows that horo-tight spheres in hyperbolic space are metric spheres. We also prove that horo-tight hyperspheres are characterized by the property that both of its total absolute horospherical curvatures attend their minimum value. We also introduce the notion of weak horo-tightness: an immersion is weak horo-tight if only one of its total absolute curvature attends its minimum. We prove a characterization theorem for weak horo-tight hyperspheres.
\end{abstract}

\section{INTRODUCTION}

What are the horo-tight immersions of spheres? We address this paper to this question proposed by Thomas E. Cecil and Patrick J. Ryan in ([9], pg 236). The notion of horo-tightness was introduced in [8], whose main subjects are tight and taut immersions into hyperbolic space. Properties of horo-tight immersions of manifolds were obtained by J. Bolton in [3].

On the other hand, in recent papers $[4,16,17,18,19]$, we discovered a new geometry in hyperbolic space which is called "Horospherical Geometry". The curvature in this geometry describes the contact of a submanifold with hyperhorospheres. It has nice properties, for instance, the Gauss-Bonnet type theorem and the Chern-Lashof type theorem hold. Therefore the horo-tightness of submanifolds in hyperbolic space is also a quite interesting notion from this point of view.

The main results in this paper are Theorems 4.3, 4.4, 4.6 and 5.1 which give several characterizations on horo-tight spheres in hyperbolic space. The results give a complete answer to the question of Cecil and Ryan. In a previous paper [5], we have considered the question of horo-tightness for circles in hyperbolic space. However, the method we used in [5] is so elementary that it cannot work for the general dimensional case. In the present paper, we complete the investigation by showing that an immersion of a sphere into hyperbolic $n$-space is horo-tight if and only if it is hyperbolic tight. For immersions in codimension higher than 1 , it follows that a horo-tight sphere is a metric sphere. There are several examples of horo-tight hyperspheres which are not metric hyperspheres even in the case when $n=2$ (i.e, horo-tight ellipses in the Poincaré disk). Therefore the situation in the codimension one case is quite different from the other cases.

One of the characterizations of horo-tight hypersphere is that it attends the minimum of the total absolute horospherical curvature. Differently from the Euclidean case, hypersurfaces in hyperbolic space always have two total absolute horospherical curvatures depending on the direction of the normals. The result is that a hypersphere is horo-tight if and only if both total absolute horospherical curvatures attend their minimum value. Therefore, we have another question : What horosphere satisfies the condition that only one of its total absolute curvature 
is minimal? As an answer to this question, we introduce the notion of weak horoconvexity and weak horo-tightness. Another result is Theorem 6.2, which gives the answer to the above new question.

After completing this work, the authors came across a preprint of E. Gallego, A. Reventós, G. Solanes and E. Teufel [11] that contains similar results. Their definition of the support map corresponds to the notion of the hyperbolic Gauss indicatrix introduced in [12]. However, they do not consider the horospherical Gauss maps $\widetilde{\mathbb{L}}^{ \pm}$nor the concept of horospherical curvature.

\section{Local Horospherical Geometry of submanifolds in Hyperbolic space}

We outline in this section the local differential geometry of submanifolds in the hyperbolic $n$-space developed in the previous papers $[12,15,16]$. We adopt, for this purpose, the model of hyperbolic $n$-space in the Minkowski $(n+1)$-space. Let $\mathbb{R}^{n+1}=\left\{\left(x_{0}, x_{1}, \ldots, x_{n}\right) \mid x_{i} \in\right.$ $\mathbb{R}(i=0,1, \ldots, n)\}$ be an $(n+1)$-dimensional vector space. For any $\boldsymbol{x}=\left(x_{0}, x_{1}, \ldots, x_{n}\right), \boldsymbol{y}=$ $\left(y_{0}, y_{1}, \ldots, y_{n}\right) \in \mathbb{R}^{n+1}$, the pseudo scalar product of $\boldsymbol{x}$ and $\boldsymbol{y}$ is defined by $\langle\boldsymbol{x}, \boldsymbol{y}\rangle=-x_{0} y_{0}+$ $\sum_{i=1}^{n} x_{i} y_{i}$. We call $\left(\mathbb{R}^{n+1},\langle\rangle,\right)$ Minkowski $(n+1)$-space and denote it by $\mathbb{R}_{1}^{n+1}$. We say that a non-zero vector $\boldsymbol{x} \in \mathbb{R}_{1}^{n+1}$ is spacelike, lightlike or timelike if $\langle\boldsymbol{x}, \boldsymbol{x}\rangle>0,\langle\boldsymbol{x}, \boldsymbol{x}\rangle=0$ or $\langle\boldsymbol{x}, \boldsymbol{x}\rangle<0$ respectively. For a vector $\boldsymbol{v} \in \mathbb{R}_{1}^{n+1}$ and a real number $c$, we define the hyperplane with pseudo normal $\boldsymbol{v}$ by $H P(\boldsymbol{v}, c)=\left\{\boldsymbol{x} \in \mathbb{R}_{1}^{n+1} \mid\langle\boldsymbol{x}, \boldsymbol{v}\rangle=c\right\}$. We call $H P(\boldsymbol{v}, c)$ a spacelike hyperplane, a timelike hyperplane or a lightlike hyperplane if $\boldsymbol{v}$ is timelike, spacelike or lightlike respectively.

We now define hyperbolic $n$-space by $H_{+}^{n}(-1)=\left\{\boldsymbol{x} \in \mathbb{R}_{1}^{n+1} \mid\langle\boldsymbol{x}, \boldsymbol{x}\rangle=-1, x_{0} \geq 1\right\}$ and de Sitter $n$-space by $S_{1}^{n}=\left\{\boldsymbol{x} \in \mathbb{R}_{1}^{n+1} \mid\langle\boldsymbol{x}, \boldsymbol{x}\rangle=1\right\}$. We have three kinds of totally umbilical hypersurfaces in $H_{+}^{n}(-1)$ which are given by the intersections of $H_{+}^{n}(-1)$ with hyperplanes. A hypersurface given by the intersection of $H_{+}^{n}(-1)$ with a spacelike hyperplane, a timelike hyperplane or a lightlike hyperplane is respectively called a hypersphere, an equidistant hypersurface or a hyperhorosphere. Especially, a hyperhorosphere is an important subject in this paper, so that we denote it by $H S(\boldsymbol{v}, c)=H_{+}^{n}(-1) \cap H P(\boldsymbol{v}, c)$, where $\boldsymbol{v}$ is a lightlike vector. We also define a set $L C_{+}^{*}=\left\{\boldsymbol{x}=\left(x_{0}, \ldots x_{n}\right) \in L C_{0} \mid x_{0}>0\right\}$, which is called the future lightcone at the origin.

In the first place, we review the results on hypersurfaces in $H_{+}^{n}(-1)$. Let $\boldsymbol{X}: U \longrightarrow$ $H_{+}^{n}(-1)$ be an embedding, where $U \subset \mathbb{R}^{n-1}$ is an open subset. We shall identify $M=\boldsymbol{X}(U)$ and $U$ through the embedding $\boldsymbol{X}$. Since $\langle\boldsymbol{X}, \boldsymbol{X}\rangle \equiv-1$, we have $\left\langle\boldsymbol{X}_{u_{i}}(u), \boldsymbol{X}(u)\right\rangle \equiv 0 \quad(i=$ $1, \ldots, n-1)$, for any $u=\left(u_{1}, \ldots u_{n-1}\right) \in U$. Therefore, we can define the spacelike unit normal $\mathbb{E}(u) \in S_{1}^{n}$. It follows that $\boldsymbol{X}(u) \pm \mathbb{E}(u) \in L C_{+}^{*}$ and hence we can define a map $\mathbb{L}^{ \pm}: U \longrightarrow L C_{+}^{*}$ by $\mathbb{L}^{ \pm}(u)=\boldsymbol{X}(u) \pm \mathbb{E}(u)$ which is called the hyperbolic Gauss indicatrix (or the lightcone dual) of $\boldsymbol{X}$. In order to define the hyperbolic Gauss-Kronecker curvature of the hypersurface $M=\boldsymbol{X}(U)$, we have shown in [12] $d \mathbb{L}^{ \pm}\left(u_{0}\right)$ is a linear transformation on the tangent space $T_{p} M$. We call the linear transformation $S_{p}^{ \pm}=-d \mathbb{L}^{ \pm}\left(u_{0}\right): T_{p} M \longrightarrow T_{p} M$ the hyperbolic shape operator of $M=\boldsymbol{X}(U)$ at $p=\boldsymbol{X}\left(u_{0}\right)$. We denote the eigenvalues of $S_{p}^{ \pm}$ by $\bar{\kappa}_{p}^{ \pm}$and the eigenvalues of $A_{p}$ by $\kappa_{i}(p)(i=1, \ldots n-1)$ which are called the hyperbolic principal curvatures. The hyperbolic Gauss-Kronecker curvature of $M=\boldsymbol{X}(U)$ at $p=\boldsymbol{X}\left(u_{0}\right)$ is defined to be $K_{h}^{ \pm}\left(u_{0}\right)=\operatorname{det} S_{p}^{ \pm}=\kappa_{1}(p) \cdots \kappa_{n-1}(p)$. Since $\boldsymbol{X}_{u_{i}}(i=1, \ldots n-1)$ are spacelike vectors, we have the Riemannian metric given by $d s^{2}=\sum_{i=1}^{n-1} g_{i j} d u_{i} d u_{j}$ on $M=\boldsymbol{X}(U)$, where $g_{i j}(u)=\left\langle\boldsymbol{X}_{u_{i}}(u), \boldsymbol{X}_{u_{j}}(u)\right\rangle$ and the hyperbolic second fundamental invariant defined by 
$\bar{h}_{i j}^{ \pm}(u)=\left\langle-\mathbb{L}_{u_{i}}^{ \pm}(u), \boldsymbol{X}_{u_{j}}(u)\right\rangle$ for any $u \in U$. In [12] the hyperbolic version of the Weingarten formula was shown and the formula $K_{h}^{ \pm}=\operatorname{det}\left(\bar{h}_{i j}^{ \pm}\right) / \operatorname{det}\left(g_{\alpha \beta}\right)$ was obtained.

In the previous paragraphs we reviewed the properties of hyperbolic Gauss indicatrices and hyperbolic Gauss-Kronecker curvatures. The original definition of the hyperbolic Gauss map introduced by Bryant [2] and Epstein [10] is given in the Poincaré ball model. Here, we introduce the corresponding definition in Minkowski model as follows: If $\boldsymbol{x}=\left(x_{0}, x_{1}, \ldots, x_{n}\right)$ is a lightlike vector, then $x_{0} \neq 0$. Therefore we have

$$
\tilde{\boldsymbol{x}}=\left(1, \frac{x_{1}}{x_{0}}, \ldots, \frac{x_{n}}{x_{0}}\right) \in S_{+}^{n-1}=\left\{\boldsymbol{x}=\left(x_{0}, x_{1}, \ldots, x_{n}\right) \mid\langle\boldsymbol{x}, \boldsymbol{x}\rangle=0, x_{0}=1\right\} .
$$

We call $S_{+}^{n-1}$ the lightcone $(n-1)$-sphere. We define a map

$$
\widetilde{\mathbb{L}}^{ \pm}: U \longrightarrow S_{+}^{n-1}
$$

by $\widetilde{\mathbb{L}}^{ \pm}(u)=\widetilde{\mathbb{L}^{ \pm}(u)}$ and call it the hyperbolic Gauss map of $\boldsymbol{X}$. Let $N_{p} M$ be the pseudo-normal space of $T_{p} M$ in $T_{p} \mathbb{R}_{1}^{n+1}$. We have the decomposition $T_{p} \mathbb{R}_{1}^{n+1}=T_{p} M \oplus N_{p} M$, so that we also have the Whitney sum $T \mathbb{R}^{n+1}=T M \oplus N M$. Therefore we have the canonical projection $\Pi$ : $T \mathbb{R}^{n+1} \longrightarrow T M$. It follows that we have a linear transformation $\Pi_{p} \circ d \widetilde{\mathbb{L}}^{ \pm}(u): T_{p} M \longrightarrow T_{p} M$ for $p=\boldsymbol{X}(u)$ by the identification of $U$ and $\boldsymbol{X}(U)=M$ via $\boldsymbol{X}$. In [16] the following formula was shown:

Proposition 2.1. Under the above notation we have the following horospherical Weingarten formula:

$$
\Pi_{p} \circ \widetilde{\mathbb{L}}_{u_{i}}^{ \pm}=-\sum_{j=1}^{n-1} \frac{1}{\ell_{0}^{ \pm}(u)}\left(\bar{h}^{ \pm}\right)_{i}^{j} \boldsymbol{X}_{u_{j}},
$$

where $\mathbb{L}^{ \pm}(u)=\left(\ell_{0}^{ \pm}(u), \ell_{1}^{ \pm}(u), \ldots, \ell_{n}^{ \pm}(u)\right)$.

We call the linear transformation $\widetilde{S}_{p}^{ \pm}=-\Pi_{p} \circ d \widetilde{\mathbb{L}}^{ \pm}$the horospherical shape operator of $M=\boldsymbol{X}(U)$. The horospherical Gauss-Kronecker curvature of $\boldsymbol{X}(U)=M$ is defined to be $\widetilde{K}_{h}^{ \pm}(u)=\operatorname{det} \widetilde{S}_{p}^{ \pm}$. It follows that we have the following relation between the horospherical Gauss-Kronecker curvature and the hyperbolic Gauss-Kronecker curvature:

$$
\widetilde{K}_{h}^{ \pm}(u)=\left(\frac{1}{\ell_{0}^{ \pm}(u)}\right)^{n-1} K_{h}^{ \pm}(u) .
$$

We remark that $\widetilde{K}_{h}^{ \pm}(u)$ is not invariant under hyperbolic motions but it is an $S O(n)$-invariant. We also remark that the notion of horospherical curvatures is independent of the choice of the model of hyperbolic space. For the purpose, we introduce a smooth function on the unit tangent sphere bundle of hyperbolic space which plays the principal role of the horospherical geometry. Let $S O_{0}(n, 1)$ be the identity component of the matrix group

$$
S O(n, 1)=\left\{g \in G L(n+1, \mathbb{R}) \mid g I_{n, 1}{ }^{t} g=I_{n, 1}\right\},
$$

where

$$
I_{n, 1}=\left(\begin{array}{c|c}
-1 & \mathbf{0} \\
\hline{ }^{t} \mathbf{0} & I_{n}
\end{array}\right) \in G L(n+1, \mathbb{R}) .
$$

It is well-known that $S O_{0}(n, 1)$ acts transitively on $H_{+}^{n}(-1)$ and the isotropic group at $p=$ $(1,0, \ldots, 0)$ is $S O(n)$ which is naturally embedded in $S O_{0}(n, 1)$. Moreover the action induces 
isometries on $H_{+}^{n}(-1)$. On the other hand, we consider a submanifold $\Delta=\{(\boldsymbol{v}, \boldsymbol{w}) \mid\langle\boldsymbol{v}, \boldsymbol{w}\rangle=$ $0\}$ of $H_{+}^{n}(-1) \times S_{1}^{n}$ and the canonical projection $\bar{\pi}: \Delta \longrightarrow H_{+}^{n}(-1)$. Let $\pi: S\left(T H_{+}^{n}(-1)\right) \longrightarrow$ $H^{n}(-1)$ be the unit tangent sphere bundle over $H_{+}^{n}(-1)$. For any $\boldsymbol{v} \in H_{+}^{n}(-1)$, we have the coordinates $\left(v_{1}, \ldots, v_{n}\right)$ of $H_{+}^{n}(-1)$ such that $\boldsymbol{v}=\left(\sqrt{v_{1}^{2}+\cdots+v_{n}^{2}+1}, v_{1}, \ldots, v_{n}\right)$. We can canonically identify $\pi: S\left(T H_{+}^{n}(-1)\right) \longrightarrow H_{+}^{n}(-1)$ with $\bar{\pi}: \Delta \longrightarrow H_{+}^{n}(-1)$. Moreover, the linear action of $S O_{0}(n, 1)$ on $\mathbb{R}_{1}^{n+1}$ induces the canonical action on $\Delta$ (i.e., $g(\boldsymbol{v}, \boldsymbol{w})=(g \boldsymbol{v}, g \boldsymbol{w})$ for any $\left.g \in S O_{0}(n, 1)\right)$. For any $(\boldsymbol{v}, \boldsymbol{w}) \in \Delta$, the first component of $\boldsymbol{v} \pm \boldsymbol{w}$ is given by

$$
v_{0} \pm w_{0}=\sqrt{v_{1}^{2}+\cdots+v_{n}^{2}+1} \pm \frac{1}{\sqrt{v_{1}^{2}+\cdots+v_{n}^{2}+1}} \sum_{i=1}^{n} v_{i} w_{i}
$$

so that it can be considered as a function on the unit tangent bundle $S\left(T H_{+}^{n}(-1)\right)$.

We now define a function

$$
\mathcal{N}_{h}: \Delta \longrightarrow \mathbb{R} ; \mathcal{N}_{h}(\boldsymbol{v}, \boldsymbol{w})=\frac{1}{v_{0}+w_{0}}
$$

We call $\mathcal{N}_{h}^{ \pm}$a horospherical normalization function on $H_{+}^{n}(-1)$. Since $v^{2}+\cdots+v_{n}^{2}+1$ and $\sum_{i=1}^{n} v_{i} w_{i}$ are $S O(n)$-invariant functions, $\mathcal{N}_{h}$ is an $S O(n)$-invariant function. Therefore, $\mathcal{N}_{h}^{ \pm}$ can be considered as a function on the unit tangent sphere bundle over the hyperbolic space $S O_{0}(n, 1) / S O(n)$ which is independent of the choice of the model space. For any embedding $\boldsymbol{X}: U \longrightarrow H_{+}^{n}(-1)\left(U \subset \mathbb{R}^{n-1}\right)$, we have the unit normal vector field $\mathbb{E}: U \longrightarrow S_{1}^{n}$, so that $(\boldsymbol{X}(u), \mathbb{E}(u)) \in \Delta$ for any $u \in U$. It follows that

$$
\widetilde{K}_{h}^{ \pm}(u)=\mathcal{N}_{h}(\boldsymbol{X}(u), \pm \mathbb{E}(u))^{n-1} K_{h}^{ \pm}(u) .
$$

The right hand side of the above equality is independent of the choice of the model space.

We now consider general submanifolds in $H_{+}^{n}(-1)$ (cf., [15]). Let $\boldsymbol{X}: U \rightarrow H_{+}^{n}(-1)$ be an embedding of codimension $(s+1)$, where $U \subset \mathbb{R}^{r}$ is an open subset $(r+s+1=n)$. We also write that $M=\boldsymbol{X}(U)$ and identify $M$ and $U$ through the embedding $\boldsymbol{X}$. Let $N_{p}(M)$ be the normal space of $M$ at $p=\boldsymbol{X}(u)$ in $\mathbb{R}_{1}^{n+1}$ and we define $N_{p}^{h}(M)=N_{p}(M) \cap T_{p} H_{+}^{n}(-1)$. Since the normal bundle $N(M)$ is trivial, we can arbitrarily choose a unit normal section $\boldsymbol{N}(u) \in S^{s}\left(N_{p}^{h}(M)\right)$. We consider the orthogonal projections $\pi^{T}: T_{p} M \oplus N_{p}^{h}(M) \longrightarrow T_{p} M$ and $\pi^{N}: T_{p} M \oplus N_{p}^{h}(M) \longrightarrow N_{p}^{h}(M)$. Let $d \boldsymbol{N}_{u}: T_{u} U \longrightarrow T_{p} M \oplus N_{p}^{h}(M)$ be the derivative of $\boldsymbol{N}$. We define that $d \boldsymbol{N}_{u}^{T}=\pi^{T} \circ d \boldsymbol{N}_{u}$ and $d \boldsymbol{N}_{u}^{N}=\pi^{N} \circ d \boldsymbol{N}_{u}$. Under the identification of $U$ and $M$, the derivative $d \boldsymbol{X}_{u}$ can be identified with the identity mapping $\mathrm{id}_{T_{p} M}$. We call the linear transformation $S_{p_{0}}(\boldsymbol{N})=-\left(\operatorname{id}_{T_{p_{0}} M}+d \boldsymbol{N}_{u_{0}}^{T}\right): T_{p_{0}} M \longrightarrow T_{p_{0}} M$ the hyperbolic $\boldsymbol{N}$ shape operator of $M=\boldsymbol{X}(U)$ at $p_{0}=\boldsymbol{X}\left(u_{0}\right)$. The hyperbolic curvature with respect to $\boldsymbol{N}$ at $p_{0}=\boldsymbol{X}\left(u_{0}\right)$ is defined to be

$$
K_{h}(\boldsymbol{N})\left(\boldsymbol{X}\left(u_{0}\right)\right)=K_{h}(\boldsymbol{N})_{p_{0}}=\operatorname{det} S_{p_{0}}(\boldsymbol{N}) .
$$

We give the following generalized hyperbolic Weingarten formula. Since $\boldsymbol{X}_{u_{i}}(i=1, \ldots r)$ are spacelike vectors, we induce the Riemannian metric (the hyperbolic first fundamental form ) $d s^{2}=\sum_{i=1}^{r} g_{i j} d u_{i} d u_{j}$ on $M=\boldsymbol{X}(U)$, where $g_{i j}(u)=\left\langle\boldsymbol{X}_{u_{i}}(u), \boldsymbol{X}_{u_{j}}(u)\right\rangle$ for any $u \in U$. We also define the hyperbolic second fundamental invariant with respect to the unit normal vector field 
$\boldsymbol{N}$ by $\bar{h}_{i j}(\boldsymbol{N})(u)=\left\langle-(\boldsymbol{X}+\boldsymbol{N})_{u_{i}}(u), \boldsymbol{X}_{u_{j}}(u)\right\rangle$ for any $u \in U$. If we define the second fundamental invariant with respect to the normal vector field $\boldsymbol{N}$ by $h_{i j}(\boldsymbol{N})(u)=-\left\langle\boldsymbol{N}_{u_{i}}(u), \boldsymbol{X}_{u_{j}}(u)\right\rangle$, then we have the following relation:

$$
\bar{h}_{i j}(\boldsymbol{N})(u)=-g_{i j}(u)+h_{i j}(\boldsymbol{N})(u),(i, j=1, \ldots, r) .
$$

Proposition 2.2. Under the above notations, we have the following horospherical (or, hyperbolic) Weingarten formula with respect to $\boldsymbol{N}$ :

$$
\pi^{T} \circ(\boldsymbol{X}+\boldsymbol{N})_{u_{i}}=-\sum_{j=1}^{r} \bar{h}_{i}^{j}(\boldsymbol{N}) \boldsymbol{X}_{u_{j}}
$$

where $\left(\bar{h}_{i}^{j}(\boldsymbol{N})\right)=\left(\bar{h}_{i k}(\boldsymbol{N})\right)\left(g^{k j}\right)$ and $\left(g^{k j}\right)=\left(g_{k j}\right)^{-1}$. It follows that the hyperbolic curvature with respect to $\boldsymbol{N}$ is given by

$$
K_{h}(\boldsymbol{N})(\boldsymbol{X}(u))=\frac{\operatorname{det}\left(\bar{h}_{i j}(\boldsymbol{N})(u)\right)}{\operatorname{det}\left(g_{\alpha \beta}(u)\right)} .
$$

Since $\left\langle-(\boldsymbol{X}+\boldsymbol{N})(u), \boldsymbol{X}_{u_{j}}(u)\right\rangle=0$, we have $\bar{h}_{i j}(\boldsymbol{N})(u)=\left\langle\boldsymbol{X}(u)+\boldsymbol{N}(u), \boldsymbol{X}_{u_{i} u_{j}}(u)\right\rangle$. Therefore the hyperbolic second fundamental invariant at a point $p_{0}=\boldsymbol{X}\left(u_{0}\right)$ depends only on $\boldsymbol{X}\left(u_{0}\right)+\boldsymbol{N}\left(u_{0}\right)$ and $\boldsymbol{X}_{u_{i} u_{j}}\left(u_{0}\right)$. By the above corollary, the hyperbolic curvature also depends only on $\boldsymbol{X}\left(u_{0}\right)+\boldsymbol{N}\left(u_{0}\right)$ and $\boldsymbol{X}_{u_{i} u_{j}}\left(u_{0}\right)$. It is independent on the choice of the normal vector field $\boldsymbol{N}$. We write $K_{h}(\boldsymbol{n})\left(\boldsymbol{X}\left(u_{0}\right)\right)$ as the hyperbolic curvature at $p_{0}=\boldsymbol{X}\left(u_{0}\right)$ with respect to $\boldsymbol{n}=\boldsymbol{N}\left(u_{0}\right)$ (i.e., $\left.K_{h}(\boldsymbol{n})\left(\boldsymbol{X}\left(u_{0}\right)\right)=K_{h}(\boldsymbol{N})\left(\boldsymbol{X}\left(u_{0}\right)\right)\right)$.

\section{Totally absolute horospherical CURVATURE}

We now consider the global properties of curvatures. We first consider the hypersurface case. Let $M$ be a closed orientable $(n-1)$-dimensional manifold and $f: M \longrightarrow$ $H_{+}^{n}(-1)$ an embedding. We consider the canonical projection $\pi: \mathbb{R}_{1}^{n+1} \longrightarrow \mathbb{R}^{n}$ defined by $\pi\left(x_{0}, x_{1}, \ldots, x_{n}\right)=\left(0, x_{1}, \ldots, x_{n}\right)$. Then we have orientation preserving diffeomorphisms $\pi \mid H_{+}^{n}(-1): H_{+}^{n}(-1) \longrightarrow \mathbb{R}^{n}$ and $\pi \mid S_{+}^{n-1}: S_{+}^{n-1} \longrightarrow S^{n-1}$. Consider the outward unit normal $\mathbb{E}$ of $f(M)$ in $H_{+}^{n}(-1)$, then we define the hyperbolic Gauss indicatrix in the global

$$
\mathbb{L}^{ \pm}: M \longrightarrow L C_{+}^{*}
$$

by

$$
\mathbb{L}^{ \pm}(p)=f(p) \pm \mathbb{E}(p)
$$

The global hyperbolic Gauss-Kronecker curvature function $\mathcal{K}_{h}: M \longrightarrow \mathbb{R}$ is then defined in the usual way in terms of the global hyperbolic Gauss indicatrix $\mathbb{L}$. We also define the hyperbolic Gauss map in the global

by

$$
\widetilde{\mathbb{L}}^{ \pm}: M \longrightarrow S_{+}^{n-1}
$$

$$
\widetilde{\mathbb{L}^{ \pm}}(p)=\widetilde{\mathbb{L}^{ \pm}(p)}
$$

We now define a global horospherical Gauss-Kronecker curvature function $\widetilde{\mathcal{K}}_{h}^{ \pm}: M \longrightarrow \mathbb{R}$ by

$$
\widetilde{\mathcal{K}}_{h}^{ \pm}(p)=\mathcal{N}_{h}(f(p), \pm \mathbb{E}(p))^{n-1} \mathcal{K}_{h}^{ \pm}(p) .
$$

In [16] the following Gauss-Bonnet type theorem for the horospherical Gauss-Kronecker curvature was shown. 
Theorem 3.1. If $M$ is a closed orientable even-dimensional hypersurface in hyperbolic $n$ space, then

$$
\int_{M} \widetilde{\mathcal{K}}_{h}^{ \pm} d \mathfrak{v}_{M}=\frac{1}{2} \gamma_{n-1} \chi(M)
$$

where $\chi(M)$ is the Euler characteristic of $M, d \mathfrak{v}_{M}$ is the volume form of $M$ and the constant $\gamma_{n-1}$ is the volume of the unit $(n-1)$-sphere $S^{n-1}$.

In order to prove the above theorem, it has been shown that $\widetilde{\mathcal{K}}_{h}^{ \pm} d \mathfrak{v}_{M}=\left(\widetilde{\mathbb{L}}^{ \pm}\right)^{*} d \mathfrak{v}_{S_{+}^{n-1}}$, where $d \mathfrak{v}_{S_{+}^{n-1}}$ is the canonical volume form of $S_{+}^{n-1}[16]$. Let $D \subset S_{+}^{n-1}$ denote the set of regular values of $\widetilde{\mathbb{L}^{ \pm}}$. Since $M$ is compact, $D$ is open and, by Sard's theorem, the complement of $D$ in $S_{+}^{n-1}$ has null measure. We define the integer valued map $\eta^{ \pm}: D \rightarrow \mathbb{E}$ by setting

$$
\eta^{ \pm}(\boldsymbol{v})=\text { the number of elements of }\left(\widetilde{\mathbb{L}^{ \pm}}\right)^{-1}(\boldsymbol{v})
$$

which turns out to be continuous. We have the following theorem.

Theorem 3.2. Let $f: M^{n-1} \rightarrow H_{+}^{n}(-1)$ be an immersion of the compact manifold $M^{n-1}$. Then

$$
\int_{M}\left|\widetilde{\mathcal{K}}_{h}^{ \pm}\right| d \mathfrak{v}_{M}=\int_{D} \eta^{ \pm}(\boldsymbol{v}) d \mathfrak{v}_{S_{+}^{n-1}}
$$

Proof. For any $\boldsymbol{v}=\widetilde{\mathbb{L}} \pm(p) \in D$, there exist a neighbourhood $V$ of $p$ in $M$ and a neighbourhood $U$ of $\boldsymbol{v}$ in $D$ such that $\widetilde{\mathbb{L}}^{ \pm} \mid V \longrightarrow U$ is a diffeomorphism. Since $\widetilde{\mathcal{K}}_{h}^{ \pm} d \mathfrak{v}_{M}=\left(\widetilde{\mathbb{L}}^{ \pm}\right)^{*} d \mathfrak{v}_{S_{+}^{n-1}}$, we have

$$
\int_{V} \widetilde{\mathcal{K}}_{h}^{ \pm} d \mathfrak{v}_{M}=\int_{U} d \mathfrak{v}_{S_{+}^{n-1}}, \text { if } \widetilde{\mathcal{K}}_{h}^{ \pm}>0 \text { on } V
$$

and

$$
\int_{V} \widetilde{\mathcal{K}}_{h}^{ \pm} d \mathfrak{v}_{M}=-\int_{U} d \mathfrak{v}_{S_{+}^{n-1}}, \text { if } \widetilde{\mathcal{K}}_{h}^{ \pm}<0 \text { on } V
$$

on $\mathrm{V}$. That is

$$
\int_{V}\left|\widetilde{\mathcal{K}}_{h}^{ \pm}\right| d \mathfrak{v}_{M}=\int_{U} d \mathfrak{v}_{S_{+}^{n-1}}
$$

We observe that the restriction of the horospherical Gauss map to $\left(\widetilde{\mathbb{L}}^{ \pm}\right)^{-1}(D)$ is a smooth (branched) covering map onto $D$ with $\eta^{ \pm}(\boldsymbol{v})$ sheets on $\boldsymbol{v} \in D$. Therefore we have

$$
\int_{M}\left|\widetilde{\mathcal{K}}_{h}^{ \pm}\right| d \mathfrak{v}_{M}=\int_{D} \eta^{ \pm}(\boldsymbol{v}) d \mathfrak{v}_{S_{+}^{n-1}}
$$

For the surface $M \subset H_{+}^{3}(-1)$, we have shown the following theorem as an application of Theorem $2.2([4])$

Theorem 3.3. Let $M^{2}$ be an embedded closed surface in $H_{+}^{3}(-1)$, then

$$
\int_{M}\left|\widetilde{\mathcal{K}}_{h}^{ \pm}\right| d \mathfrak{a}_{M} \geq 2 \pi(4-\chi(M)) .
$$


We remark that the right hand side of the inequality will be much more complicated if we consider a hypersurface $M \subset H_{+}^{n}(-1)$. Actually we need some information on the Betti numbers of $M$ and the volume of the unit sphere $S^{n-1}$. However, we have the following rough estimate:

Theorem 3.4. Let $f: M \longrightarrow H_{+}^{n}(-1)$ be an embedding from a closed orientable manifold with dimension $n-1$. Then we have

$$
\int_{M}\left|\widetilde{\mathcal{K}}_{h}^{ \pm}\right| d \mathfrak{v}_{M} \geq \gamma_{n-1}
$$

where $\gamma_{n-1}$ is the volume of the unit sphere $S_{+}^{n-1}$. The equality holds if and only if $\widetilde{\mathbb{L}}^{ \pm}$is bijective on the regular values.

Proof. It is enough to show that $\widetilde{\mathbb{L}}^{ \pm}$is surjective. In [16] it has been defined the horospherical height function $h_{v}^{h}$ on $M$ for any $\boldsymbol{v} \in S_{+}^{n-1}$ (see also $\S 4$ ). It has also shown that $p \in M$ is a critical point of $h_{v}^{h}$ if and only if $\boldsymbol{v}=\widetilde{\mathbb{L}}^{+}(p)$ or $\boldsymbol{v}=\widetilde{\mathbb{L}}^{-}(p)$. For any $\boldsymbol{v} \in S_{+}^{n-1}$, there exists a maximum point $p_{0}$ and a minimum point $q_{0}$ of the horospherical height function $h_{v}^{h}$ on the compact manifold $M$. These points are critical points of $h_{v}^{h}$, so that $\boldsymbol{v}=\widetilde{\mathbb{L}}^{+}\left(p_{0}\right)$ or $\boldsymbol{v}=\widetilde{\mathbb{L}}^{-}\left(p_{0}\right)$ (and $\boldsymbol{v}=\widetilde{\mathbb{L}}^{+}\left(q_{0}\right)$ or $\boldsymbol{v}=\widetilde{\mathbb{L}}^{-}\left(q_{0}\right)$.) It is enough to show that $\widetilde{\mathbb{L}}^{+}\left(p_{0}\right) \neq \widetilde{\mathbb{L}}^{+}\left(q_{0}\right)$. Suppose that $\widetilde{\mathbb{L}}^{+}\left(p_{0}\right)=\widetilde{\mathbb{L}}^{+}\left(q_{0}\right)$. We define a function $\widetilde{h}_{v}: H_{+}^{n}(-1) \longrightarrow \mathbb{R}$ by $\widetilde{h}_{v}(\boldsymbol{x})=\langle\boldsymbol{x}, \boldsymbol{v}\rangle$. It follows that $\widetilde{h}_{v} \circ f(p)=h_{v}^{h}(p)$. We now distinguish two cases.

(i) If $\boldsymbol{v}=\widetilde{\mathbb{L}}^{+}\left(p_{0}\right)$, then we have $\boldsymbol{v}=\widetilde{\mathbb{L}}^{+}\left(q_{0}\right)$. We now consider the geodesic from $f\left(q_{0}\right)$ directed by $-\mathbb{E}\left(q_{0}\right)$, parametrized by

$$
\gamma_{q_{0}}(t)=\cosh t f\left(q_{0}\right)-\sinh t \mathbb{E}\left(q_{0}\right)
$$

Then we have

$$
\begin{aligned}
\frac{d \tilde{h}_{v} \circ \gamma_{q_{0}}}{d t}(t) & =\left\langle\frac{d \boldsymbol{\gamma}_{q_{0}}}{d t}(t), \boldsymbol{v}\right\rangle=\left\langle\frac{d \boldsymbol{\gamma}_{q_{0}}}{d t}(t), \tilde{\mathbb{L}}^{+}\left(q_{0}\right)\right\rangle \\
& =\left\langle\sinh t f\left(q_{0}\right)-\cosh t \mathbb{E}\left(q_{0}\right), \frac{1}{\ell_{0}\left(q_{0}\right)}\left(f\left(q_{0}\right)+\mathbb{E}\left(q_{0}\right)\right)\right\rangle \\
& =\frac{1}{\ell_{0}\left(q_{0}\right)}(-\sinh t-\cosh t)=\frac{1}{\ell_{0}\left(q_{0}\right)}(\sinh (-t)-\cosh (-t))<0
\end{aligned}
$$

where $f(p)+\mathbb{E}(p)=\mathbb{L}^{+}(p)=\left(\ell_{0}(p), \ell_{1}(p), \ldots, \ell_{n}(p)\right) \in L C_{+}^{*}$, so that $\widetilde{h}_{v} \circ \gamma(t)$ is strictly decreasing. Since $q_{0}$ is the minimum point of $h_{v}^{h}$ and $f\left(q_{0}\right)=\gamma_{q_{0}}(0), \gamma_{q_{0}}(t) \notin f(M)$ for any $t>0$. Since $H_{+}^{n}(-1)$ is a complete Riemannian manifold, $\gamma_{q_{0}}(t)$ can be expanded for any large $t>0$. It follows that there exists a positive real number $\tau$ that $\gamma_{q_{0}}(\tau)$ is in the outside of $f(M)$ (i.e, the unbounded component of $H_{+}^{n}(-1) \backslash f(M)$ ).

On the other hand, since $\mathbb{E}\left(q_{0}\right)$ is the outward unit normal of $f(M)$ at $f\left(q_{0}\right),-\mathbb{E}\left(q_{0}\right)$ is the inward unit normal, so that there exists a sufficiently small $\varepsilon>0$ such that $\gamma_{q_{0}}(\varepsilon)$ is in the inside of $f(M)$. By the Jordan-Brouwer separation theorem, there exists a real number $t_{0}>0$ such that $\gamma_{q_{0}}\left(t_{0}\right) \in f(M)$. This is a contradiction.

(ii) If $\boldsymbol{v}=\widetilde{\mathbb{L}}^{-}\left(p_{0}\right)$, then we also consider the geodesic from $f\left(p_{0}\right)$ directed by $-\mathbb{E}\left(p_{0}\right)$, parametrized by

$$
\gamma_{p_{0}}(t)=\cosh t f\left(p_{0}\right)-\sinh t \mathbb{E}\left(p_{0}\right)
$$


Then we have

$$
\begin{aligned}
\frac{d \widetilde{h}_{v} \circ \boldsymbol{\gamma}_{p_{0}}}{d t}(t) & =\left\langle\frac{d \boldsymbol{\gamma}_{p_{0}}}{d t}(t), \boldsymbol{v}\right\rangle=\left\langle\sinh t f\left(p_{0}\right)-\cosh t \mathbb{E}\left(p_{0}\right), \frac{1}{\ell_{0}\left(p_{0}\right)}\left(f\left(p_{0}\right)-\mathbb{E}\left(p_{0}\right)\right)\right\rangle \\
& =\frac{1}{\ell_{0}\left(p_{0}\right)}(-\sinh t+\cosh t)>0
\end{aligned}
$$

so that $\widetilde{h}_{v} \circ \gamma(t)$ is strictly increasing. Since $p_{0}$ is the maximum point of $h_{v}^{h}$ and $f\left(p_{0}\right)=\gamma_{p_{0}}(0)$, $\gamma_{p_{0}}(t) \notin f(M)$ for any $t>0$. By exactly the same reason as in the case (i), there exists a real number $t_{0}>0$ such that $\gamma_{q_{0}}\left(t_{0}\right) \in f(M)$. This is a contradiction.

Eventually, we have $\widetilde{\mathbb{L}}^{+}\left(p_{0}\right) \neq \widetilde{\mathbb{L}}^{+}\left(q_{0}\right)$. This means that $\boldsymbol{v}=\widetilde{\mathbb{L}}^{+}\left(p_{0}\right)=\widetilde{\mathbb{L}}^{-}\left(q_{0}\right)$ or $\boldsymbol{v}=$ $\widetilde{\mathbb{L}}^{-}\left(p_{0}\right)=\widetilde{\mathbb{L}^{+}}\left(q_{0}\right)$. Therefore $\widetilde{\mathbb{L}}^{+}$and $\widetilde{\mathbb{L}}^{-}$are both surjective, so that we have the required inequality.

By Theorem 3.2, the equality holds if and only if $\eta^{ \pm} \equiv 1$. This condition is equivalent to the condition that $\widetilde{\mathbb{L}}^{ \pm}$is bijective on the regular values.

We now define the total absolute horospherical curvature for an embedding $f: M \longrightarrow$ $H_{+}^{n}(-1)$ from a closed orientable manifold with dimension $n-1$ by

$$
\tau_{h}^{ \pm}(f ; M)=\frac{1}{\gamma_{n-1}} \int_{M}\left|\widetilde{\mathcal{K}}_{h}^{ \pm}\right| d \mathfrak{v}_{M} .
$$

On the other hand, we consider general submanifolds in $H_{+}^{n}(-1)$. Let $M$ be a compact $r$-dimensional manifold and $f: M^{r} \rightarrow H_{+}^{n}(-1)$ denotes an immersion of codimension $(s+1)$. Let $\nu^{1}(M)$ denote the unitary normal bundle of the immersion $f$, i.e.:

$$
\nu^{1}(M)=\left\{(p, \xi) ; \xi \in N_{p}^{h}(M) \text { and }\langle\xi, \xi\rangle=1\right\} .
$$

The horospherical Gauss map $\widetilde{\mathbb{L}}: \nu^{1}(M) \rightarrow S_{+}^{n-1}$ of the immersion $f: M^{s} \rightarrow H_{+}^{n}(-1)$ is defined by the following commutative diagram

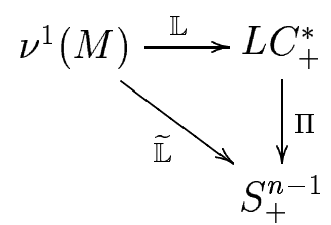

where $\mathbb{L}: \nu^{1}(M) \rightarrow L C_{+}^{*} ; \mathbb{L}(p, \xi)=f(p)+\xi$ is called the hyperbolic Gauss indicatrix of the immersion $f$ and $\Pi(\boldsymbol{v})=\widetilde{\boldsymbol{v}}$. The horospherical Gauss map lead us to a curvature in the framework of horospherical geometry. Let $T_{(\boldsymbol{x}, \boldsymbol{n})} \nu^{1}(M)$ be the tangent space of $\nu^{1}(M)$ at $(\boldsymbol{x}, \boldsymbol{n})$. We have the canonical identification $T_{(\boldsymbol{x}, \boldsymbol{n})} \nu^{1}(M)=T_{\boldsymbol{x}} M \oplus T_{\boldsymbol{n}} S^{s} \subset T_{\boldsymbol{x}} M \oplus$ $N_{\boldsymbol{x}} M=T_{\boldsymbol{x}} \mathbb{R}_{1}^{n+1}$, where $N_{\boldsymbol{x}} M$ is the normal vector space of $M$ at $\boldsymbol{x}$ in $\mathbb{R}_{1}^{n+1}$. Let $P$ : $\widetilde{\mathbb{L}}^{*} T \mathbb{R}_{1}^{n+1}=T \nu^{1}(M) \oplus \mathbb{R}^{2} \rightarrow T \nu^{1}(M)$ be the canonical projection. It follows that we have a linear transformation

$$
P_{\widetilde{\mathbb{L}}(\boldsymbol{x}, \boldsymbol{n})} \circ d \widetilde{\mathbb{L}}: T_{(\boldsymbol{x}, \boldsymbol{n})} \nu^{1}(M) \rightarrow T_{(\boldsymbol{x}, \boldsymbol{n})} \nu^{1}(M) .
$$

The horospherical curvature with respect to $\boldsymbol{n}$ at $\boldsymbol{x}$ is defined to be

$$
\widetilde{K}_{h}(\boldsymbol{x}, \boldsymbol{n})=\operatorname{det}\left(P_{\widetilde{\mathbb{L}}(\boldsymbol{x}, \boldsymbol{n})} \circ(-d \widetilde{\mathbb{L}})\right) .
$$


In [4] we have shown that

$$
\widetilde{K}_{h}(\boldsymbol{x}, \boldsymbol{n})(p)=\mathcal{N}_{h}^{n-1}(\boldsymbol{x}, \boldsymbol{n}) K_{h}(\boldsymbol{n})(f(p)) .
$$

and

$$
\left(\widetilde{\mathbb{L}}^{*} d \mathfrak{v}_{S_{+}^{n-1}}\right)_{(\boldsymbol{x}, \boldsymbol{n})}=\left|\widetilde{K}_{h}(\boldsymbol{x}, \boldsymbol{n})\right| d \mathfrak{v}_{\nu^{1}(M)} .
$$

The total absolute horospherical curvature of the immersion $f$ is defined by

$$
\tau_{h}(f ; M)=\frac{1}{\gamma_{n-1}} \int_{\nu^{1}(M)} \widetilde{\mathbb{L}}^{*} \sigma .
$$

It follows from the above formula that we have

$$
\tau_{h}(f ; M)=\frac{1}{\gamma_{n-1}} \int_{\nu^{1}(M)}\left|\widetilde{K}_{h}(\boldsymbol{x}, \boldsymbol{n})\right| d \mathfrak{v}_{\nu^{1}(M)},
$$

In [4] we have shown the following Chern-Lashof type theorem.

Theorem 3.5. Let $f: M^{r} \rightarrow H_{+}^{n}(-1)$ be an immersion of the compact manifold $M$. Then

(1) $\tau_{h}(f ; M) \geqslant \gamma(M) \geqslant 2$;

(2) if $\tau_{h}(f ; M)<3$ then $M$ is homeomorphic to the sphere $S^{r}$.

It has been posed the following question in [4]:

Question 3.6. How is the geometry of $f(M) \subset H_{+}^{n}(-1)$ if $\tau_{h}(f ; M)=2$ ?

In this paper we also give an answer to this question.

Remark 3.7. If $r=n-1$, then $\nu^{1}(M)$ is a double covering over $M$, so that $\widetilde{\mathbb{L}}(p, \pm \mathbb{E}(p))=$ $f(p) \pm \mathbb{E}(p)=\widetilde{\mathbb{L}^{ \pm}}(p)$ (i.e., $\left.\mathbb{L}(p, \pm \mathbb{E}(p))=\mathbb{L}^{ \pm}(p)\right)$. Therefore, we have the following weaker inequality as a corollary of Theorem 3.5:

$$
\tau_{h}^{+}(f ; M)+\tau_{h}^{-}(f ; M)=\frac{1}{\gamma_{n-1}}\left(\int_{M}\left|\widetilde{\mathcal{K}}_{h}^{+}\right| d \mathfrak{v}_{M}+\int_{M}\left|\widetilde{\mathcal{K}}_{h}^{-}\right| d \mathfrak{v}_{M}\right)=\tau_{h}(f ; M) \geq 2 .
$$

In $\S 6$ we give one of the examples of curves in $H_{+}^{2}(-1)$ such that

$$
\int_{M}\left|\widetilde{\mathcal{K}}_{h}^{+}\right| d \mathfrak{v}_{M} \neq \int_{M}\left|\widetilde{\mathcal{K}}_{h}^{-}\right| d \mathfrak{v}_{M} .
$$

\section{Horo-tight imMERSions OF SPHERES With HIGHER CODIMENSION}

We first define two families of functions

$$
H^{h}: M \times S_{+}^{n-1} \longrightarrow \mathbb{R}
$$

by $H^{h}(p, \boldsymbol{v})=\langle f(p), \boldsymbol{v}\rangle$ and

$$
H^{d}: M \times S_{1}^{n} \longrightarrow \mathbb{R}
$$

by $H^{d}(p, \boldsymbol{v})=\langle f(p), \boldsymbol{v}\rangle$. We call $H^{h}$ a horospherical height functions family and $H^{d}$ a de Sitter height functions family on $f: M \longrightarrow H_{+}^{n}(-1)$. Each $h_{v_{0}}^{h}(p)=H^{h}\left(p, \boldsymbol{v}_{0}\right)$ for fixed $\boldsymbol{v}_{0} \in S_{+}^{n-1}$ (respectively, $h_{v_{0}}^{d}(p)=H^{d}\left(p, \boldsymbol{v}_{0}\right)$ for fixed $\left.\boldsymbol{v}_{0} \in S_{1}^{n}\right)$ is called a horospherical height function (respectively, de Sitter height function). We denote the Hessian matrix of the horospherical height function $h_{v_{0}}^{h}$ at $p_{0} \in M$ by $\operatorname{Hess}\left(h_{v_{0}}^{h}\right)\left(p_{0}\right)$. We say that the critical point $p \in M$ of $h_{v_{0}}^{h}$ is non-degenerate if $\operatorname{det} \operatorname{Hess}\left(h_{v_{0}}^{h}\right)\left(u_{0}\right) \neq 0$. We say that a function $f: M \longrightarrow \mathbb{R}$ is nondegenerate if $f$ has only non-degenerate critical points. An immersion $f: M \longrightarrow H_{+}^{n}(-1)$ is 
said to be hyperbolic tight ( $H$-tight for short) if every non-degenerate de Sitter height function $h_{v}^{d}$ has the minimum number of critical points required by the Morse inequalities. We also say that $f: M \longrightarrow H_{+}^{n}(-1)$ is horospherical tight (horo-tight for short) if every non-degenerate horospherical height function $h_{v}^{h}$ has the minimum number of critical points required by the Morse inequalities.

Remark 4.1. In [7] a function $L_{h}: H_{+}^{n}(-1) \longrightarrow \mathbb{R}$ has been defined to be $L_{h}(p)=\ln \left(-h_{v}^{h}(p)\right)$ which is called the distance function from $p$ to the hyperhorosphere $H S(\boldsymbol{v},-1)$ for $\boldsymbol{v} \in S^{n-1}$. Therefore the minimum of $L_{h}$ corresponds to the maximum of $h_{v}^{h}$ (i.e., the minimum of $-h_{v}^{h}$ ). We do not use this fact in this section, but it will be needed in $\S 6$.

A set $X \subset H_{+}^{n}(-1)$ is convex if for any pair of points in $X$ the geodesic segment joining them is contained in $X$. Every hyperhorosphere $\mathcal{H}$ in $H_{+}^{n}(-1)$ is the boundary of a closed convex region of $H_{+}^{n}(-1)$. These convex subsets are called $h$-convex. We say that a submanifold (or, an immersion) $f: M \rightarrow H_{+}^{n}(-1)$ is horospherical convex (horo-convex for short) if for any $p \in M$, one of the h-convex sets determined by its tangent hyperhorosphere at $f(p)$ contains $f(M)$ entirely. One of the interesting properties of Euclidean tightness is the two piece property with respect to hyperplanes [1]. There is an analogous notion in hyperbolic space. We say that $f: M \longrightarrow H_{+}^{n}(-1)$ has the two-piece property with respect to hyperhorospheres if both of $f^{-1}(\mathfrak{h})$ and $f^{-1}\left(\overline{H_{+}^{n}(-1) \backslash \mathfrak{h}}\right)$ are connected for every closed h-convex set $\mathfrak{h}$ in $H_{+}^{n}(-1)$, where $\bar{X}$ denotes the closure of $X$ in $H_{+}^{n}(-1)$. By definition, we have the following simple proposition.

Proposition 4.2. If an immersion $f: M \longrightarrow H_{+}^{n}(-1)$ is horo-tight, then it has the two-piece property with respect to hyperhorospheres.

The main results in this section are the following.

Theorem 4.3. Let $f: S^{r} \rightarrow H_{+}^{n}(-1)$ be an immersion. Then $f$ is horo-tight if and only if $f$ is $H$-tight.

We remark that the above theorem gives an answer to the question of Cecil and Ryan. For $n>r+1$ this theorem is a corollary of the following characterization of horo-tight embeddings of spheres in higher codimension.

Theorem 4.4. Let $f: S^{r} \rightarrow H_{+}^{r+k}(-1), k>1$ be an immersion. Then $f$ is horo-tight if and only if $f$ embeds $S^{r}$ as an $r$-dimensional metric sphere.

The following properties of horo-tight immersions of manifolds into hyperbolic space can be found in [3].

Theorem 4.5. [Bolton, Theorem 1] Let $f: M \rightarrow H_{+}^{n}(-1)$ be an immersion of a compact manifold into the hyperbolic space. The following conditions are equivalent:

(i) $M$ is homeomorphic to a sphere and $f(M)$ is horo-tight.

(ii) $f(M)$ lies in only one side of any tangent hyperhorosphere.

(iii) The horospherical Gauss map $\widetilde{\mathbb{L}}: \nu^{1}(M) \longrightarrow S_{+}^{n-1}$ takes every regular value exactly twice.

We can give an answer to Question 3.6 as follows.

Theorem 4.6. Let $f: M \rightarrow H_{+}^{n}(-1)$ be an immersion of a compact manifold into the hyperbolic space. Then $M$ is homeomorphic to a sphere and $f(M)$ is horo-tight if and only if

$$
\tau_{h}(f ; M)=2 .
$$


Proof. In order to prove Theorem 3.5, we have introduced the following function in [4]: Let $D \subset S_{+}^{n-1}$ denote the set of regular values of $\widetilde{\mathbb{L}}$. Since $M$ is compact, $D$ is open and, by Sard's theorem, the complement of $D$ in $S_{+}^{n-1}$ has null measure. We define the integer valued map $\kappa: D \rightarrow \mathbb{N}$ by setting

$$
\kappa(\boldsymbol{v})=\text { number of elements of } \tilde{\mathbb{L}}^{-1}(\boldsymbol{v}),
$$

which turns out to be continuous. Moreover, we have shown that

$$
\tau_{h}(f ; M)=\frac{1}{\gamma_{n-1}} \int_{D} \kappa(\boldsymbol{v}) d \mathfrak{v}_{S_{+}^{n-1}} .
$$

It follows that $\tau_{h}(f ; M)=2$ if and only if $\kappa(\boldsymbol{v})=2$. This condition is equivalent to the condition (iii) in Theorem 4.5.

We now restrict the arguments to the case of immersions in codimension bigger than 1 .

Proposition 4.7. Let $f: M^{r} \rightarrow H_{+}^{n}(-1), n>r+1$ be an immersion of a compact manifold into the hyperbolic space. If one of the above conditions (i) to (iii) (and hence all of them) of Theorem 4.5 holds, then $f(M)$ lies in one hyperhorosphere.

Proof. Suppose that $M$ is homeomorphic to a sphere and $f$ is horo-tight. Then there exists a point $p \in S^{r}$ such that $-h_{v^{+}}^{h}$ has the maximum at $p$ where $\boldsymbol{v}^{+}=\widetilde{\mathbb{L}}(p, \xi)$. In this case, $f\left(S^{r}\right)$ lies in the convex side of one of the tangent hyperhorosphere at $f(p)$ whose normal is $\xi$. This means that $f\left(S^{r}\right)$ lies in the concave side of another tangent hyperhorosphere at $f(p)$ whose normal is $-\xi$ (i.e., determined by $\left.\boldsymbol{v}^{-}=\widetilde{\mathbb{L}}(p,-\xi)\right)$. On the unit normal space $\nu^{1}\left(S^{r}\right)_{p}$ at $p$, there exists a path $\gamma: I \longrightarrow \nu^{1}\left(S^{r}\right)_{p}$ such that $\gamma(0)=\xi$ and $\gamma(1)=-\xi$. We can consider the Gauss image $\boldsymbol{v}^{\gamma(t)}=\widetilde{\mathbb{L}}(p, \gamma(t))$. Since $n>r+1$, there exists a one-parameter family of tangent hyperhorospheres of $f\left(S^{r}\right)$ at $p$ such that $f\left(S^{r}\right)$ lies in the convex side of the tangent hyperhorosphere determined by $\boldsymbol{v}^{\gamma(0)}=\boldsymbol{v}^{+}$and lies in the concave side of the tangent hyperhorosphere determined by $\boldsymbol{v}^{\gamma(1)}=\boldsymbol{v}^{-}$. Therefore, there exists a point $t_{0} \in I$ such that $f\left(S^{r}\right)$ lies on the tangent hyperhorosphere determined by $\boldsymbol{v}^{\gamma\left(t_{0}\right)}$. Otherwise it contradicts the above proposition.

We are now prepared to prove Theorem 4.4.

Proof. If $f\left(S^{r}\right)$ lies on a hyperhorosphere and $f$ is horo-tight, then $f\left(S^{r}\right)$ is taut in a hyperhorosphere (in the Euclidean sense). In fact, we remark that the intersection of two hyperhorospheres is a $n$-2-dimensional metric sphere in both hyperhorospheres. On the other hand, any $n$-2-dimensional metric sphere in a hyperhorosphere is given by the intersection of the hyperhorosphere and another hyperhorosphere. Therefore, the horo-spherical two piece property implies the spherical two piece property on a hyperhorosphere. Therefore, the horotight sphere in a hyperhorosphere is a taut sphere in a hyperhorosphere in the Euclidean sense (or the spherical sense). It follows from a result of Cecil [6] that a taut sphere in Euclidean space is a metric sphere, hence $f\left(S^{r}\right)$ is a metric sphere. This completes the proof of the theorem.

From the above result and Theorem 4.2 in [8], it follows that an immersion $f: S^{r} \rightarrow$ $H_{+}^{n}(-1), n>r+1$ is horo-tight if and only if it is $H$-tight. We postpone the proof of case $n=r+1$ in Theorem 4.3 for the next section. 


\section{Horo-Tightness AND HoroconVEXity OF HYPERSPHERES}

In this section we consider the characterization of spheres in hyperbolic space which attend the minimum of the total absolute horospherical curvature. We first consider the case of hypersurfaces in hyperbolic space. Let $f: M \longrightarrow H_{+}^{n}(-1)$ be an embedding from an $(n-1)$ dimensional manifold. In the first place, we recall that the minimum for the total absolute curvature of a hypersphere in Euclidean space $\mathbb{R}^{n}$ is 1 and this minimum is attained precisely when the image is the convex hypersphere. Moreover, for codimension one embeddings of spheres in Euclidean spaces, the property of attending the minimum of the total absolute curvature is equivalent to the notion of tightness. We would like to obtain a similar result for the image of hyperspheres in hyperbolic space.

The main theorem in this section is the following.

Theorem 5.1. For an immersion $f: S^{n-1} \longrightarrow H_{+}^{n}(-1)$, the following conditions are equivalent:

(1) $f$ is horo-convex.

(2) $\tau_{h}^{+}\left(f ; S^{n-1}\right)=\tau_{h}^{-}\left(f ; S^{n-1}\right)=1$.

(3) $\tau_{h}\left(f ; S^{n-1}\right)=2$.

(4) Both mappings $\widetilde{\mathbb{L}}^{+}$and $\widetilde{\mathbb{L}}^{-}$are bijective on the regular values.

(5) $f$ is horo-tight.

(6) $f$ is H-tight.

In order to prove the above theorem, we need some preparations. Let $\boldsymbol{v}$ be an arbitrary point in $H_{+}^{n}(-1)$. Then $H P(\boldsymbol{v}, 0)$ is a spacelike hyperplane on which $\langle$,$\rangle restricts to an Euclidean$ metric. Let $D_{v}^{n}$ be the unit disc centered at the origin in $\operatorname{HP}(\boldsymbol{v}, 0)$. Then the stereographic projection with pole $-\boldsymbol{v}$ is the diffeomorphism

$$
P_{v}: H_{+}^{n}(-1) \longrightarrow D_{v}^{n}
$$

given by

$$
P_{v}(\boldsymbol{x})=-\boldsymbol{v}+\frac{\boldsymbol{x}+\boldsymbol{v}}{1-\langle\boldsymbol{x}, \boldsymbol{v}\rangle} .
$$

If we choose $\boldsymbol{v}=(1,0, \ldots, 0)$, we denote $D_{v}^{n}=D^{n}$ and $P_{v}=P$. We can induce the metric $g_{v}$ on $D_{v}^{n}$ through $P_{v}$ from $H_{+}^{n}(-1)$, so that $\left(D_{v}^{n}, g_{v}\right)$ gives a model of hyperbolic space. We call it the Poincaré ball model of hyperbolic space. We observe that $P_{v}$ is conformal into Euclidean space $H P(\boldsymbol{v}, 0)$, so that a submanifold of $H_{+}^{n}(-1)$ is umbilical if and only if its image under $P_{v}$ is umbilical in $H P(\boldsymbol{v}, 0)$ in the Euclidean sense. For an immersion $f: M \longrightarrow H_{+}^{n}(-1)$, we say that $P_{v} \circ f$ is $E$-convex (respectively, E-tight) if $P_{v} \circ f$ is convex (tight) in $H P(\boldsymbol{v}, 0)$ in the Euclidean sense.

Before we give a proof of Theorem 5.1, we remark that a horo-tight immersion of a sphere is an embedding.

Proposition 5.2. Let $f: S^{n-1} \longrightarrow H_{+}^{n}(-1)$ be a horo-tight immersion. Then

(1) For any $\boldsymbol{v} \in S_{+}^{n-1}$, the horospherical height function $h_{v}^{h}$ has exactly two critical values.

(2) $f$ is an embedding.

Proof. (1) We define a subset $W \subset S_{+}^{n-1}$ by

$$
\begin{aligned}
W=\left\{\boldsymbol{v} \in S_{+}^{n-1} \mid\right. & h_{v}^{h} \text { has a critical point other than } \\
& \text { the global maximum and the global minimum }\} .
\end{aligned}
$$


By continuity, $W$ is an open subset of $S_{+}^{n-1}$. By Sard's theorem, the set of regular values of the hyperbolic Gauss map $\widetilde{\mathbb{L}}^{ \pm}$is dense, so that the horospherical height function $h_{v}^{h}$ is non-degenerate for almost all $v \in S^{n-1}$. By definition such a function has exactly two critical points. It follows that $W$ must be empty.

(2) It is enough to show that $f$ is injective. Let us assume that there exists $p \neq q \in S^{n-1}$ such that $f(p)=f(q)$. Let $\boldsymbol{v} \in S_{+}^{n-1}$ such that $p$ is a critical point of $h_{v}^{h}$. By (1), $p$ is the maximum point or the minimum point of $h_{v}^{h}$. It follows that $q$ is also a critical point of $h_{v}^{h}$. This contradicts the assumption that $h_{v}^{h}$ has only one maximum and one minimum points.

Proof of Theorem 5.1. Suppose that the condition (2) holds. Then we have

$$
\tau_{h}\left(f ; S^{n-1}\right)=\tau_{h}^{+}\left(f ; S^{n-1}\right)+\tau_{h}\left(f ; S^{n-1}\right)=2 .
$$

This means that (2) implies (3). It follows from Theorem 4.6 that $f$ is horo-tight, so that (3) implies (5). By Theorem 3.4 and Proposition 5.2, we may assume that $f$ is an embedding and (5) implies (2). We have shown that the conditions (2), (3) and (5) are equivalent.

Since $\nu^{1}\left(S^{n-1}\right)$ is a double covering on $S^{n-1}$ and $\widetilde{\mathbb{L}}(p, \pm \xi)=\widetilde{\mathbb{L}}^{ \pm}(p)$, the condition (4) is equivalent to the condition (iii) in Theorem 4.5. Moreover, suppose that $f$ is not horo-convex. Then there exists a point $p \in S^{n-1}$ and a lightlike vector $\boldsymbol{v} \in S_{+}^{n-1}$ such that one of the tangent hyperhorosphere of $f(M)$ at $p$ determined by $\boldsymbol{v} \in S_{+}^{n-1}$ separates $f\left(S^{n-1}\right)$ into at least two parts. By the assertion (ii) in Theorem 4.5, this means that $f$ is not horo-tight. It is trivial that the condition (ii) in Theorem 4.5 implies that $f$ is horo-convex. It follows that the equivalences $(1) \Leftrightarrow(4) \Leftrightarrow(5)$ follows from Theorem 4.5.

The implication $(6) \Rightarrow(5)$ is a particular case of Theorem 5.1 of [8]. In order to prove that (5) implies (6), we remark that $f$ is H-tight if and only if $\Phi \circ P \circ f$ is E-tight for every isometry $\Phi$ of the Poincaré ball model $\left(D^{n}, g\right)$ (cf. [8], Corollary 4.2). Let us assume that $\Phi \circ P \circ f$ is not E-tight for some isometry $\Phi$ of $D^{n}$. It follows that $\alpha=\Phi \circ P \circ f$ is a non-convex hypersphere. Hence there exists a tangent hyperplane $H P(f(p), c)$ at some point $p$ such that none of its closed half-hyperplanes contains $\Phi \circ P \circ f\left(S^{n-1}\right)$ entirely. Now let $H S(f(p))$ be a tangent hyperhorosphere of $\Phi \circ P \circ f$ at $p$ which passes through the interior of $\Phi \circ P \circ f\left(S^{n-1}\right)$. See Figure 1 in the case when $n=2$, where the two tangent horocycles of $\Phi \circ P \circ f$ at $p$ are drawn. Actually we can show the existence of such a hyperhorosphere as follows: At the point $f(p)$, there exist exactly two tangent hyperhorospheres. If both of them do not pass through the interior of $\Phi \circ P \circ f\left(S^{n-1}\right)$, then $p$ is the minimum point of both of $-h_{v^{ \pm}}^{h}$, where $\boldsymbol{v}^{ \pm}=\widetilde{\mathbb{L}}^{ \pm}(p)$. This means that both of $\boldsymbol{v}^{ \pm}$are the outward lightlike normal of $\Phi \circ P \circ f\left(S^{n-1}\right)$ at $p$. It follows that $\Phi \circ P \circ f\left(S^{n-1}\right)$ is not normally orientable. This is a contradiction. Therefore we have the tangent hyperhorosphere of $\Phi \circ P \circ f$ at $p$ which passes trough the interior of $\Phi \circ P \circ f\left(S^{n-1}\right)$.

Let $\boldsymbol{v} \in S_{+}^{n-1}$ be the center of this tangent hyperhorosphere (i.e., $H S(f(p))=H_{+}^{n}(-1) \cap$ $H(\boldsymbol{v}, c))$. In this case $\boldsymbol{v}=\widetilde{\mathbb{L}}^{+}(p)$ or $\boldsymbol{v}=\widetilde{\mathbb{L}}^{-}(p)$. Without loss of generality, we assume that $\boldsymbol{v}=\widetilde{\mathbb{L}}^{+}(p)$. It follows that $p$ is a critical point of $h_{v}^{h}$ which is neither the maximum point nor the minimum point. This is a contradiction with Proposition 5.2. Therefore (5) implies (6). This completes the proof of Theorem 5.1. 


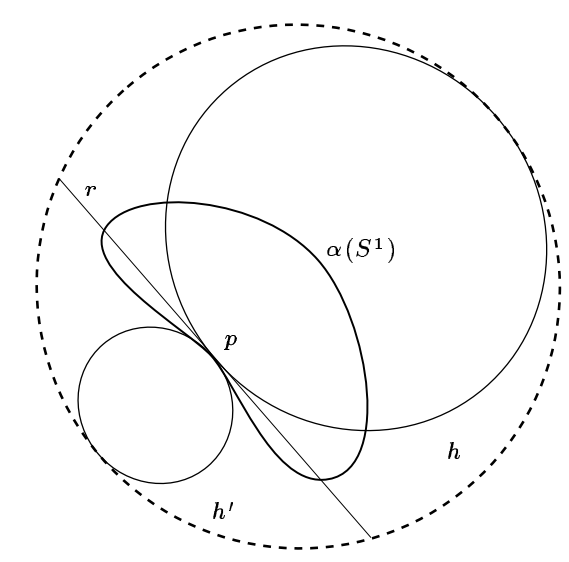

FIGURE 1. Horo-tightness implies convexity.

\section{WEAK HOROCONVEXITY AND WEAK HORO-TIGHTNESS FOR HYPERSPHERES}

We now exhibit a non-horoconvex closed curve $\gamma$ with the property that only one of its total absolute curvature attends the minimum 1. In order to proceed the calculation, we consider a unit speed hyperbolic plane curve $\gamma: S^{1} \rightarrow H_{+}^{2}(-1)$. In [13] it has been shown the Frenet-Serret type formula:

$$
\left\{\begin{aligned}
\gamma^{\prime}(s) & =\boldsymbol{t}(s) \\
\boldsymbol{t}^{\prime}(s) & =\boldsymbol{\gamma}(s)+\kappa_{g}(s) \boldsymbol{e}(s) \\
\boldsymbol{e}^{\prime}(s) & =-\kappa_{g}(s) \boldsymbol{t}(s)
\end{aligned}\right.
$$

were $\boldsymbol{t}$ is the unit tangent vector, $\boldsymbol{e}$ is the normal vector defined in the same way as in the general case and $\kappa_{g}(s)$ is the geodesic curvature of $\gamma$ in $H_{+}^{2}(-1)$ which is given by

$$
\kappa_{g}(s)=\operatorname{det}\left(\boldsymbol{\gamma}(s), \boldsymbol{t}(s), \boldsymbol{t}^{\prime}(s)\right) .
$$

We have $\mathbb{L}^{ \pm}=\gamma \pm \boldsymbol{e}$. If we fix the following parametrization of the lightlike circle:

$$
S_{+}^{1}=\{(1, \cos \theta, \sin \theta) \mid 0 \leqslant \theta \leqslant 2 \pi\},
$$

then the horospherical curvatures are

$$
\widetilde{\kappa}_{h}^{ \pm}(s)=\mathcal{N}_{h}(\gamma(s), \pm \boldsymbol{e}(s))\left( \pm \kappa_{g}(s)-1\right) .
$$

Therefore we have

$$
\tau_{h}^{ \pm}\left(\boldsymbol{\gamma}, S^{1}\right)=\frac{1}{2 \pi} \int_{S^{1}}\left|\mathcal{N}_{h}(\boldsymbol{\gamma}(s), \pm \boldsymbol{e}(s))\left( \pm \kappa_{g}(s)-1\right)\right| d s \geq 1 .
$$

We consider the following example.

Example 6.1. For simplicity we shall give its parametrization in the Poincaré disk. For the embedded circle in $D^{2}$, we choose the orientation of the circle that the bounded region is always located in the left hand side of the curve. In this case we can easily show that $-\mathbb{E}$ 
corresponds to the outward unit normal of the embedded circle. We consider an (Euclidean) ellipse in the Poincaré disk given by

$$
\gamma: S^{1} \rightarrow D^{2} ; \gamma(t)=(0.4 \cos t, 0.1 \sin t)
$$

We remark that $\tilde{\mathbb{L}}^{-}$is bijective on the regular values if $\left\|\frac{d}{d t} \widetilde{\mathbb{L}}^{-}(t)\right\| \neq 0$ for any $t \in S^{1}$, and this is the case for the curve $\gamma$, as we can see from Figure 2 . Therefore, we have $\tau_{h}^{-}\left(\gamma ; S^{1}\right)=1$. From Figure 3, we see that $\gamma$ is cut into two pieces by one of its tangent horocycles and thus it is non-horoconvex. This means that $\tau_{h}^{+}\left(\gamma ; S^{1}\right)>1$ by Theorem 3.5.

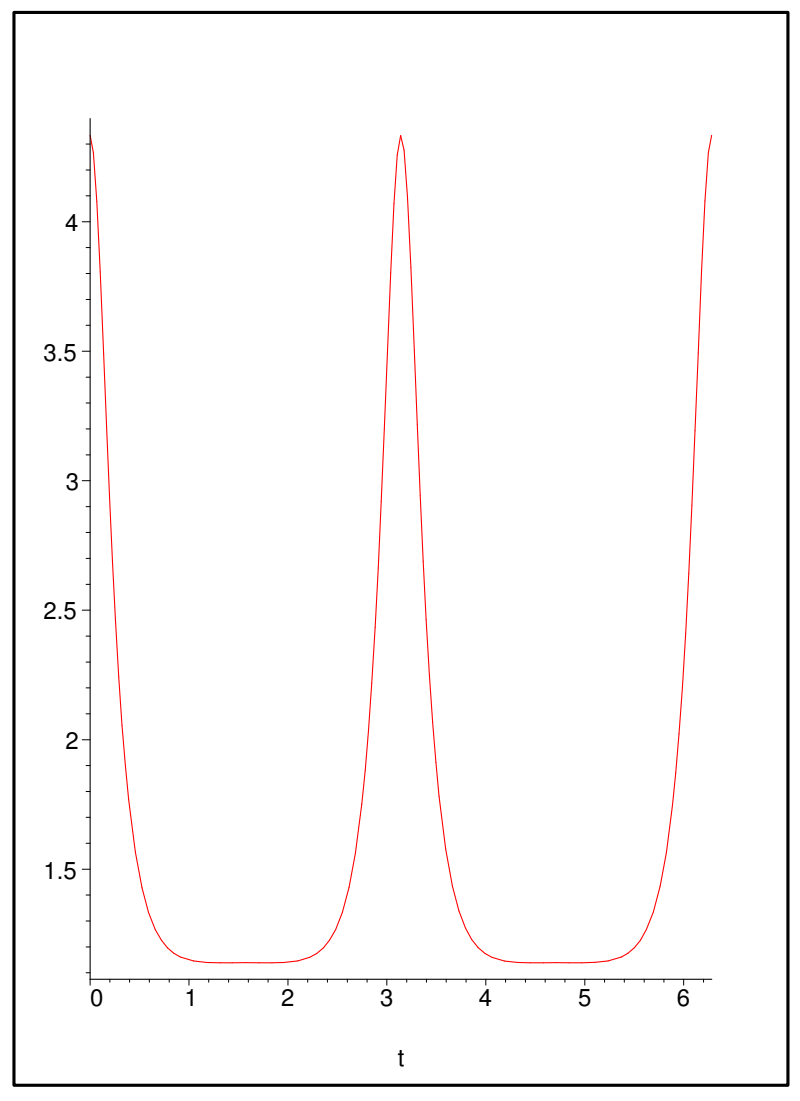

FigURE 2. Graphic of $\left\|\frac{d}{d t} \widetilde{\mathbb{L}}^{-}(t)\right\|$

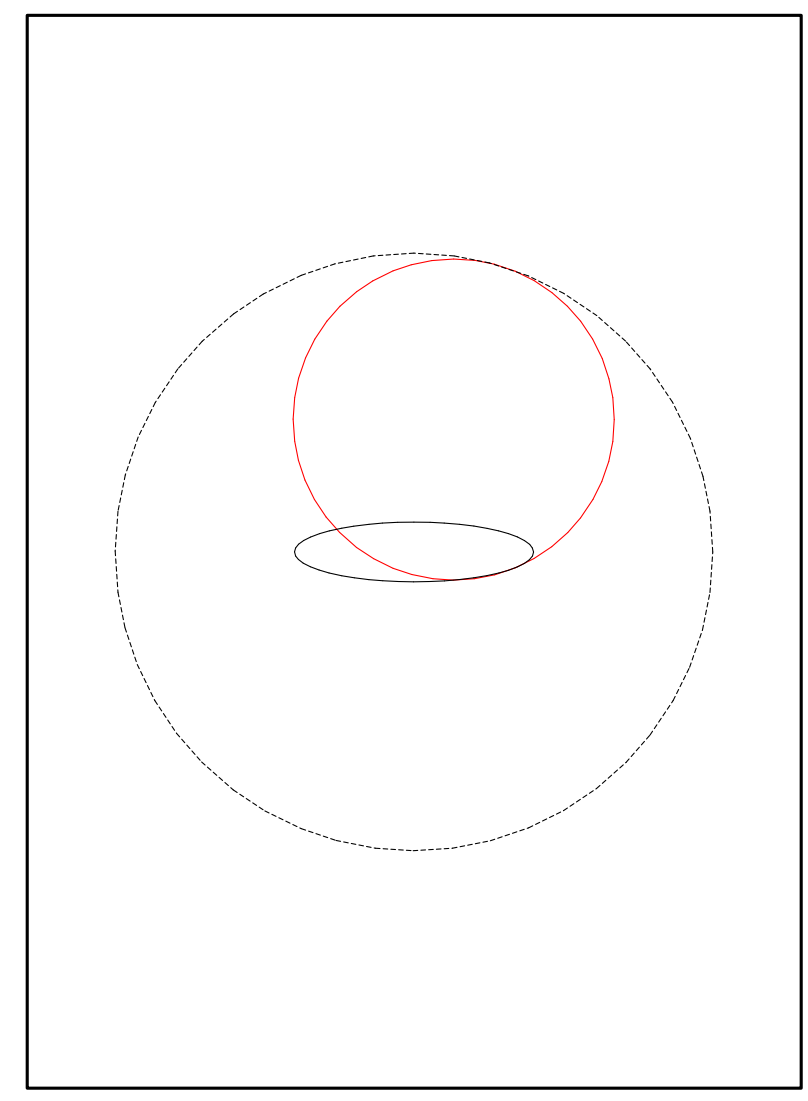

FiguRE 3. Separating horocycle

The above example suggests the following new question : What are the hyperspheres in hyperbolic space satisfying the condition that only one of their total absolute curvatures attends the minimum value 1 ?

For an embedding $f: S^{n-1} \longrightarrow H_{+}^{n}(-1)$, we choose an orientation such that $d P \circ \mathbb{E}$ is an inward unit normal of $P \circ f\left(S^{n-1}\right)$. Here $P: H_{+}^{n}(-1) \longrightarrow D^{n}$ is the stereographic projection onto the Poincaré ball $D^{n}$. We remark that $-d P \circ \mathbb{E}$ is the outward unit normal along $P \circ$ $f\left(S^{n-1}\right)$. In this case we have the tangent hyperhorosphere defined by $H S(\boldsymbol{v}, c)=H_{+}^{n}(-1) \cap$ $H P(\boldsymbol{v}, c)$, where $\boldsymbol{v}=\widetilde{\mathbb{L}}^{-}(p)$ and $c=\langle\boldsymbol{v}, f(p)\rangle$ at any $p \in S^{n-1}$. We denote the tangent hyperhorosphere by $H S^{-}\left(f\left(S^{n-1}\right), p\right)$ and call it the outward tangent hyperhorosphere at $p \in$ $S^{n-1}$. We say that $f$ is weak horoconvex if $f\left(S^{n-1}\right)$ completely belongs to the complement of 
the interior of the h-convex set determined by the outward tangent hyperhorosphere at any point $p \in S^{n-1}$.

Let $p \in S^{n-1}$ be a critical point of $h_{v}^{h}$. Then we have exactly two tangent hyperhorospheres at $f(p)$ such that $\boldsymbol{v} \in S_{+}^{n-1}$ is the lightlike normal vector. A critical point $p \in S^{n-1}$ of $h_{v}^{h}$ is said to be outward if $\boldsymbol{v}$ is an outward lightlike normal vector (i.e., $\boldsymbol{v}=\widetilde{\mathbb{L}}^{-}(p)$ ). We say that $f: S^{n-1} \longrightarrow H_{+}^{n}(-1)$ is weak horo-tight if every non-degenerate horospherical height function $h_{v}^{h}$ has only one minimum of $-h_{v}^{h}$ as the outward critical point.

Theorem 6.2. For an embedding $f: S^{n-1} \longrightarrow H_{+}^{n}(-1)$, we choose an orientation such that $\mathbb{E}$ is the unit inward normal vector field along $f$, then the following conditions are equivalent:

(1) $f$ is weak horoconvex.

(2) $\tau_{h}^{-}\left(f ; S^{n-1}\right)=1$.

(3) $\widetilde{\mathbb{L}}^{-}$is bijective on the regular values.

(4) $f$ is weak horo-tight.

If we choose the opposite orientation, the above conditions are changed into those for $\tau_{h}^{+}\left(f ; S^{n-1}\right)$ and $\widetilde{\mathbb{L}}^{+}$.

Proof. The arguments for the proof are the almost parallel to those of the proofs of Theorem 4.5 and Theorem 5.1. By the same arguments as in these theorems, (2),(3) and (4) are equivalent.

We now prove that (1) implies (4). Suppose that $f$ is not weak horo-tight. Then there exists a non-degenerate horospherical height function $h_{v}^{h}$ which has at least two outward critical points. If necessary, under a small perturbation of $\boldsymbol{v} \in S_{+}^{n-1}$, all critical values of $h_{v}^{h}$ are different. It follows that one of them is not the minimum of $-h_{v}^{h}$. This means that the tangent outward hyperhorosphere of $f\left(S^{n-1}\right)$ at $p$ determined by the outward lightlike vector $\boldsymbol{v} \in S_{+}^{n-1}$ locally separates $f\left(S^{n-1}\right)$ into at least two parts. Therefore $f$ is not weak horoconvex.

We also give the proof of the converse assertion. Under the assumption that $f$ is weak horo-tight, suppose that $f$ is not weak horoconvex. Then there exists a point $p \in S^{n-1}$ and an outward lightlike vector $\boldsymbol{v} \in S_{+}^{n-1}$ of $f\left(S^{n-1}\right)$ such that the tangent outward hyperhorosphere of $f\left(S^{n-1}\right)$ at $p$ determined by $\boldsymbol{v} \in S_{+}^{n-1}$ locally separates $f\left(S^{n-1}\right)$ into at least two parts. This means that $p$ is an outward critical point of $h_{v}^{h}$ which is not the minimum point of $-h_{v}^{h}$. If $p$ is non-degenerated critical point, it contradicts the assumption that $f$ is weak horo-tight. If $p$ is degenerate critical point, under a small perturbation of $\boldsymbol{v} \in S_{+}^{n-1}$, we have a nondegenerate outward critical point $p^{\prime}$ which is not the minimum point of $-h_{v}^{h}$. This contradicts the assumption that $f$ is horo-tight. This completes the proof of Theorem 6.2.

Remark 6.3. We can consider the notion of Euclidean convexity in the Poincaré ball model. Of course this notion is not a hyperbolic invariant, but it is an $S O(n)$-invariant. By definition, if $f$ is horo-convex then $P \circ f$ is E-convex. Moreover, if $P \circ f$ is E-convex, then it is weak horoconvex. However the example shows that the weak horoconvexity does not imply the convexity in the Euclidean sense (see Figure 1).

\section{REFERENCES}

[1] T. F. Banchoff, Tightly embedded 2-dimensional polyhedral manifolds, Amer. J. Math. 87 (1965) $462-472$.

[2] R. L. Bryant, Surfaces of mean curvature one in hyperbolyc space, Théorie de variétés minimales et applications (Palaiseau, 1983-1984), Astèrisque No. 154-155, (1987), 12, 321-347, 353 (1988). 
[3] J. Bolton, Tight immersions into manifolds without conjugate points, Quart. J. Math. Oxford (2), 33 (1982), 159-167.

[4] M. Buosi, S. Izumiya and M. A. Ruas, Total absolute horospherical curvature of submanifolds in Hyperbolic space, to appear in Advances in Geometry

[5] M. Buosi, S. Izumiya and M. A. Ruas, Horo-tight circles in hyperbolic space, preprint (2008).

[6] T. E. Cecil,A characterization of metric spheres in hyperbolic space by Morse theory, Tohoku Math. J.. 26(1974), 341-351.

[7] T. E. Cecil and P. J. Ryan, Distance functions and umbilic submanifolds of hyperbolic space, Nagoya Math. J., 74 (1979), 67-75.

[8] T. E. Cecil and P. J. Ryan, Tight ant taut immersions into hyperbolic space, J. London Math. Soc., 19 (1979), 561-572.

[9] T. E. Cecil and P. J. Ryan, Tight ant taut immersions of manifolds, Research Notes in Mathematics, 107 (1985), Pitman.

[10] C. L. Epstein, The hyperbolic Gauss map and quasiconformal reflections. J. Reine Angew. Math., 372 (1986), 96-135

[11] E. Gallego, A. Reventós, G. Solanes and E. Teufel, Horospheres in hyperbolic geometry, preprint.

[12] S. Izumiya, D. Pei, T. Sano, Singularity of the hyperbolic Gauss maps, Proceedings of the London Mathematical Society. 86 (2003) 485-512.

[13] S. Izumiya, D. Pei, T. Sano and E. Torii, Evolutes of hyperbolic plane curves, Acta. Math. Sinica. 20 (2004) 543-550.

[14] S. Izumiya, D. Pei and M. Takahashi, Singularities of evolutes of hypersurfaces in hyperbolic space, Proceedings of Edinburgh Mathematical Society (2004) 47, 131-153.

[15] S. Izumiya, D. Pei, M. D. R. Fuster, and M. Takahashi, The horospherical geometry of submanifolds in hyperbolic space, J. London Math. Soc., (2) 71 (2005) 779-800.

[16] S. Izumiya, and M. C. Romero Fuster, The horospherical Gauss-Bonnet type theorem in hyperbolic space, Journal of Mathematical Society of Japan. 58 (2006) 965-984.

[17] S. Izumiya, Horospherical geometry in hyperbolic space, in Noncommutativity and SingularitiesProceedings of French-Japanese symposia held at IHES in 2006, to appear in Advanced Studies in Pure Mathematics.

[18] S. Izumiya, K. Saji and M. Takahashi, Horospherical flat surfaces in Hyperbolic 3-space, Hokkaido University preprint series $\mathbf{8 3 8},(2007)$

[19] C. Takizawa and K. Tsukada, Horocyclic surfaces in hyperbolic 3-space, preprint (2008).

[20] J. A. Thorpe, Elementary Topics in Differential Geometry, Undergraduate Texts in Mathematics, (1979), Springer.

Universidade Federal do Espírito Santo, Departamento de Matemática Av. Fernando FerRARI, 514, Goiabeiras, 29075-910, VitóRIa, ES, BraziL.

Department of Mathematics, Hokkaido University, Sapporo 060-0810, Japan.

Instituto de Ciências Matemáticas e de Computação, Universidade de São Paulo, Departamento de Matemática, Caixa Postal 668, 13560-970, São Carlos, SP, Brazil.

E-mail address, Marcelo Buosi: pmarpceplo@yahoo.com.br

E-mail address, Shyuichi Izumiya: izumiya@math.sci.hokudai.ac.jp

E-mail address, Maria Aparecida Soares Ruas: maasruas@icmc.usp.br 